Safti, Hisham El; Bonakdar, Lisham; Oumeraci, Hocine A Hybrid 2D-3D CFD Model System for Offshore Pile Groups Subject to Wave Loading

Zur Verfügung gestellt in Kooperation mit / Provided in Cooperation with: Kuratorium für Forschung im Küsteningenieurwesen (KFKI)

Verfügbar unter / Available at:

https://hdl.handle.net/20.500.11970/99429

Vorgeschlagene Zitierweise / Suggested citation:

Safti, Hisham El; Bonakdar, Lisham; Oumeraci, Hocine (2014): A Hybrid 2D-3D CFD Model System for Offshore Pile Groups Subject to Wave Loading. In: Lehfeldt, Rainer; Kopmann, Rebekka (Hg.): ICHE 2014. Proceedings of the 11th International Conference on Hydroscience \& Engineering, September 28 October 2, 2014, Hamburg, Germany. Karlsruhe: Bundesanstalt für Wasserbau. S. 177-184. 


\title{
A Hybrid 2D-3D CFD Model System for Offshore Pile Groups Subject to Wave Loading
}

\author{
H. El Safti, L. Bonakdar \& H. Oumeraci \\ Leichtweiss-Institute for Hydraulic Engineering and Water Resources, Dept. of Hydromechanics and \\ Coastal Engineering, TU Braunschweig, Braunschweig, Germany
}

\begin{abstract}
A hybrid 2D-3D CFD model is developed for studying water wave loads acting on a pile in a pile group. In the hybrid model approach, a one-way link is established between a model for the farfield and another for studying the (local) fluid-structure interaction phenomenon. For this study, the farfield is considered as a 2D incompressible Navier-Stokes multiphase solver for proper reproduction of phase-focused waves produced in physical experiments. The near-field model is a multiphase 3D CFD model that utilizes compressible Navier-Stokes equations to enhance simulation of entrapped air compressibility effects during breaking wave impact on structures. Both models use the Volume-Of-Fluid (VOF) method to capture the air-water interface. An overlap zone is introduced to both models, in which fluid kinematics and surface elevation are sampled from the far-field model and introduced via a relaxation function to the overlap zone in the near-field model. In the 3D model, the use of a relaxation approach provides absorption for reflected waves from the structure. Further, a procedure is outlined to achieve/enhance the 3D model convergence. This is necessary in case of development of artificial high velocities at water-air interface at the end of a short overlap (relaxation) zone for wave inlet (or near the boundary if only a wave inlet boundary condition is considered). The model system is developed using the OpenFOAM ${ }^{\circledR}$ framework. The overlap zone is implemented as an extension to the waves 2 Foam toolbox (Jacobsen et al., 2012).
\end{abstract}

Keywords: CFD, OpenFOAM ${ }^{\circledR}$, Pile groups, Wave loading, Focused waves

\section{INTRODUCTION}

Piles have always played an important role in supporting fixed marine structures. These structures range from offshore oil and gas (fixed) platforms to maritime transportation infrastructure, such as sea bridges, piers and jetties. Most commonly, marine structures would be supported by a pile group rather than a single pile. For closely spaced piles, the effect of wave-pile group interaction is more obvious, as piles produce interference effects that change the flow around the piles. Consequently, wave loads on a pile in a pile group may vary significantly from those exerted on a single pile in isolation.

Experimental studies were carried out for studying different wave loads on a pile in a pile group. The physical experiments include large-scale tests in the Large Wave Flume (GWK) in Hanover (Sparboom and Oumeraci, 2006; Bonakdar and Oumeraci, 2012). The experiments were aimed at enhancing the knowledge about the effect of wave-pile group interaction on wave loads on a pile in a group. These experiments are used for validation of the numerical model at hand.

The most important drawbacks of the available studies are (i) the lack of deeper understanding of the processes associated with the interaction of breaking/non-breaking waves and pile groups and (ii) the lack of reliable formulae for the prediction of wave forces on a slender pile within a pile group in different arrangements. Within the WaPiGS project, additional scale-experiments were carried out to meet the aforementioned challenges. Further, and in complement to physical experiments, a numerical model is necessary to extend the testing conditions (e.g. pile group configurations) to further improve the data base of results. 
Additionally, the numerical model provides an excellent tool for further understanding of physical processes involved in the problem by providing flexible and comprehensive output options. Nonetheless, a numerical model must be validated (e.g. against physical experiments) before being approved for the aforementioned purposes.

While considerable effort has been expended in numerical simulation of wave forces on an isolated vertical cylinder, much less attention has been paid to the influence of neighbouring cylinders on wave loading, although many offshore structures are founded on groups of cylindrical piles in different arrangements. While some methods are available for calculating wave loads on a pile in a pile-array, they do not provide an insight into the relevant physics. They are also neither reliable nor convenient for practical engineering applications. It is evident that computational fluid dynamics (CFD) models are able to assess the effects of the wave-pile group interaction on the hydrodynamic forces on any one support, as well as providing more comprehensive results for the understanding of relevant processes involved. Further, CFD models constitute powerful tools for assisting design purposes and decisions making.

\section{THE 2D-3D HYBRID CFD MODEL APPROACH}

The model is implemented in the Eulerian multiphase (Volume-Of-Fluid) solver; interFoam. The fluid continuity equation (mass balance) is modified for the incompressible multiphase solver to account for fluid compressibility:

$\nabla . \mathbf{U}+\frac{1}{K_{f}} \frac{\partial p}{\partial t}=0$

Where the fluid bulk modulus $K_{f}$ is calculated based on the phase fraction $(\gamma)$ as:

$\frac{1}{K_{f}}=\frac{\gamma}{K_{w}}+\frac{(1-\gamma)}{K_{a}}$

For $K_{w}=2200 \mathrm{MPa}$ is the bulk modulus of pure water, the bulk modulus of air $K_{a}=p+p_{0}$; where $p$ is the fluid pressure and $p_{0}=0.101 \mathrm{MPa}$ is the atmospheric pressure.

The hybrid 2D-3D model system consists of three main parts:

1. A 2D wave flume (without any structure present) with a moving piston (dynamic mesh) wave maker, extending for a long distance before the position of the structure (i.e. similar to the Large Wave Flume, GWK) in order to properly simulate wave-wave interaction needed to construct phase focused (rogue) waves. The domain extends for enough distance after the position of the structure to allow for wave absorption,

2. Linking utilities to sample the data from the $2 \mathrm{D}$ model and to introduce them to the $3 \mathrm{D}$ model inside an overlap zone using a relaxation function. The linking utilities are equipped with interpolation and mapping capabilities to ensure independence of time and space discretization of both the $2 \mathrm{D}$ and the $3 \mathrm{D}$ models,

3. A small 3D wave basin with inlet and outlet boundaries close to the structure with two relaxation zones for the generation (overlap zone) and absorption of waves. The structure is modelled in the domain between both relaxation zones.

The outline of the developed 2D-3D hybrid CFD model system is illustrated in Figure 1. The procedure for using the hybrid 2D-3D CFD model system is as follows:

1. Prepare the $2 \mathrm{D}$ flume and provide positions for velocity probes and wave gauges inside the overlap zone in the 2D flume

2. Run the $2 \mathrm{D}$ model to simulate each wave condition

3. Use the linking utilities to prepare probed velocities and water surface data for use as input in the 3D model

4. Prepare the geometry (and mesh) for the 3D model for each structural configuration, starting at the overlap zone

5. Run the 3D model for each combination of structural configuration and wave conditions

In Figure 2, a sketch is given for the dual domains used in the hybrid model system with illustration of system components. 


\begin{tabular}{|c|c|c|}
\hline Far-field (2D) model & & Near-field (3D) model \\
\hline $\begin{array}{l}\text { * A 2D numerical wave flume } \\
\text { (without any structure) to generate } \\
\text { Focused (rogue) waves by } \\
\text { implementing paddle motions } \\
\text { (dynamic mesh) } \\
\text { ^ LES turbulence modelling } \\
\text { * Wave absorption by waves2Foam } \\
\text { * Input motion from physical ex- } \\
\text { periments or calculated externally } \\
\text { by a Gaussian wave packet } \\
\text { approach } \\
\text { * Simulations as many as the studied }\end{array}$ & $\begin{array}{l}\text { * Sampling surface elevation and } \\
\text { velocity probes in overlap zone } \\
\text { from 2D model } \\
\text { * Transformation of sampled data } \\
\text { to prepare as input for the 3D } \\
\text { model } \\
\text { * Space and time linear inter- } \\
\text { Polation between sampled } \\
\text { values and input to 3D model } \\
\text { * Arelaxation function to } \\
\text { Introduce wave input in the 3D } \\
\text { model inside the overlap zone }\end{array}$ & $\begin{array}{l}\text { * Reduced 3D numerical wave basin } \\
\text { (domain) consisting of different } \\
\text { structural configurations } \\
\text { * LES turbulence modelling } \\
\text { * Wave generation by a new } \\
\text { implemented waveTheory class } \\
\text { hybrid2D3D as an extension to } \\
\text { the waves2Foam toolbox } \\
\text { * Wave absorption by waves2Foam } \\
\text { * Simulations as many as the } \\
\text { studied wave conditions for each } \\
\text { structural configuration }\end{array}$ \\
\hline
\end{tabular}

Figure 1. Outline of the developed 2D-3D hybrid CFD model system

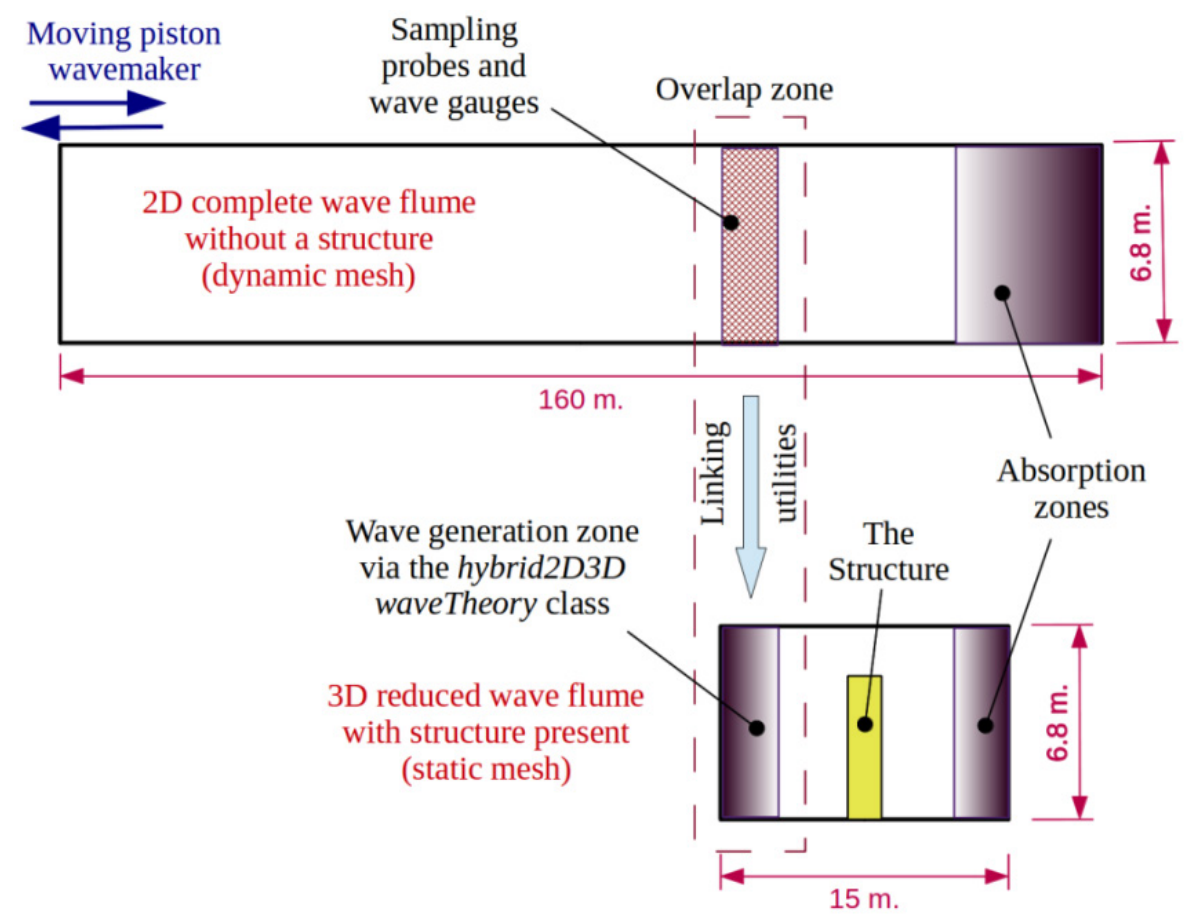

Figure 2. Sketch of the dual domains used for the 2D-3D hybrid model system with the linking utilities

\section{MODEL VALIDATION}

An extensive large-scale model tests have been carried out in the LargeWave Flume (GWK) of the Coastal Research Center (FZK) in Hanover, Germany. The tested pile groups were categorized in three different arrangements including side by side, tandem and staggered arrangements. Overall, 15 pile configurations with gaps of up to three times the pile diameter were tested. The measured pile, made of steel with a diameter $0.324 \mathrm{~m}$., was installed like a cantilever pile (Figure 3 ) in a water depth of $4.26 \mathrm{~m}$. in the middle of the large wave flume at a distance of $104.69 \mathrm{~m}$. from the wave paddle.

The physical experiments in the Large Wave Flume (GWK) in Hanover (Sparboom and Oumeraci, 2006; Bonakdar and Oumeraci, 2012) are used for the validation of the 2D-3D CFD hybrid model system. First, 2D CFD simulations of phase-focused (freak) waves are carried out similar to their generation in the physical experiments. Second, the wave conditions obtained from the $2 \mathrm{D}$ simulations are used as input for 
3D simulations for wave loads on a single pile and two different pile group configurations. The 2D simulations cover the total length of the Large Wave Flume (GWK). The model employs dynamic mesh capabilities of OpenFOAM ${ }^{\circledR}$ to model a moving piston wave maker to produce phase-focused waves as they are generated in physical experiments,
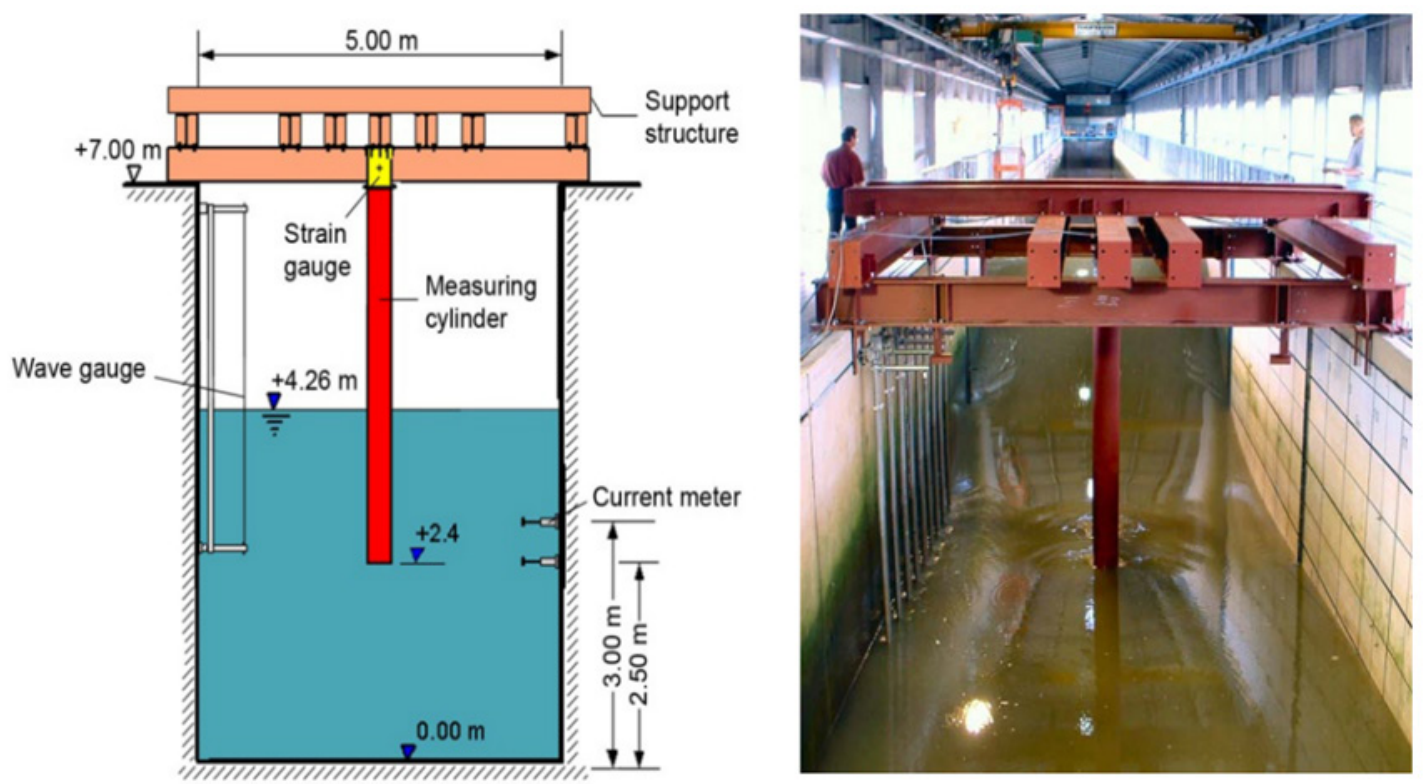

Figure 3. GWK model set-up for a single pile

Five conditions are used in the Large Wave Flume (GWK) for focusing waves in five different positions of the flume. The different positions were simulated successfully. The results of one condition (for focusing waves $103 \mathrm{~m}$. away from the wave maker) are presented herein. Results from two different wave gauges are presented in Figure 4 (finest mesh size $0.01388 \mathrm{~m}$.).

As shown in Figure $4 b$, at the peak of the focused wave the computed water surface from the numerical simulation $(2.6 \mathrm{~m}$. after the structure $)$ is less than the measured from the physical experiments. This can be most likely due to the manner in which the water surface elevation is computed by the waves 2 Foam toolbox. The water surface elevation (at any given wave gauge) is calculated as the integration of the phase fraction along the wave gauge at a prescribed height interval multiplied by the interval. As wave breaking initializes and causes tongues to form and consequently high air content air-water mixtures (Figure $4 \mathrm{~b}$ ), the implemented method becomes inaccurate as it tends to superpose water and eliminate effect of air pockets and hence does not capture the highest point attained by water. A different implementation of numerical wave gauges (e.g. based on highest point with interpolated phase fraction of 0.5 ) should provide a better agreement between numerical results and physical measurements, after initialization of wave breaking.

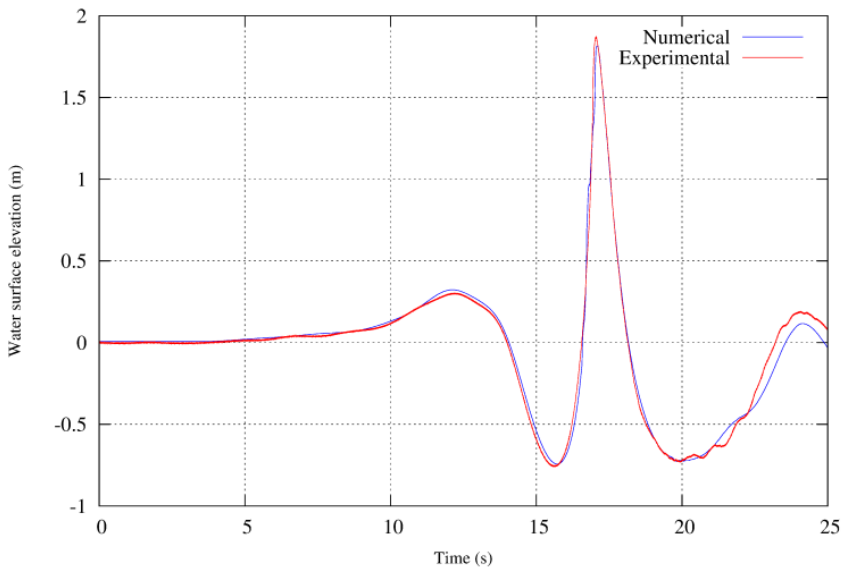

(a)

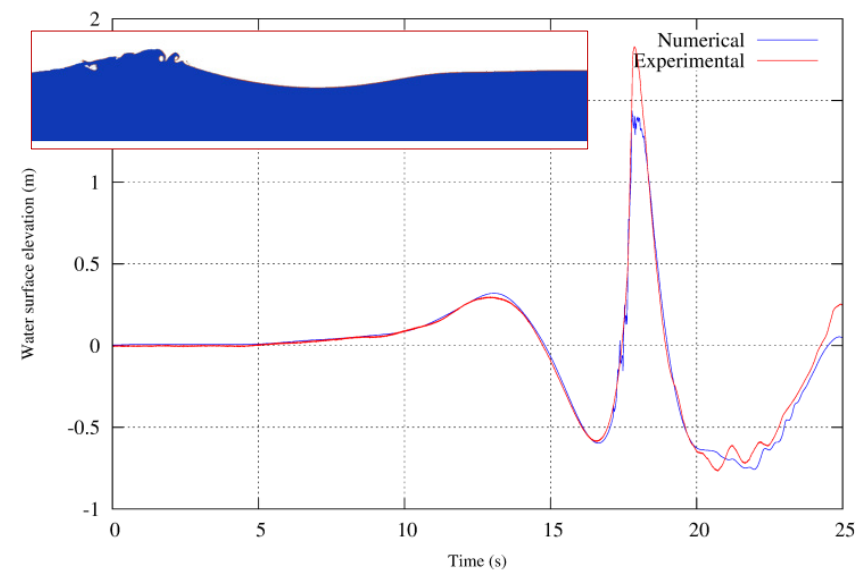

(b)

Figure 4. Water surface for phase-focused waves $25.54 \mathrm{~m}$. before and $2.6 \mathrm{~m}$. after the position of the structure 


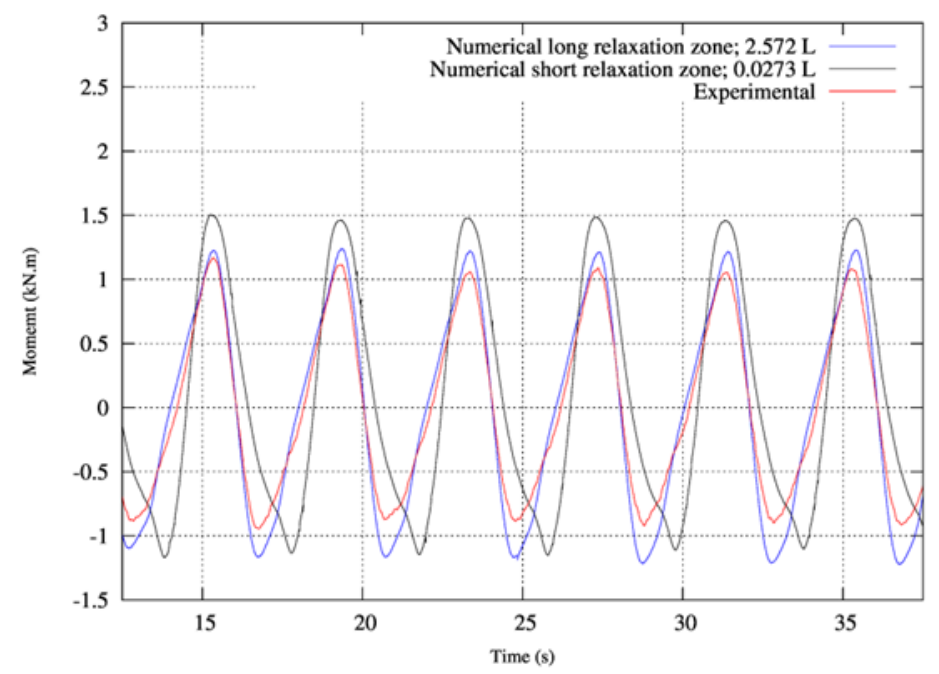

Figure 5. Wave induced inline moment on the top of a single pile for regular Stokes fifth order waves, $\mathrm{T}=4 \mathrm{~s} ., \mathrm{H}=0.8 \mathrm{~m}$ and $\mathrm{d}=4.26 \mathrm{~m}$. (finest mesh size $0.055 \mathrm{~m}$.)

To reduce the size of the 3D model further, the symmetry of the problem is considered to reduce the model size by half via introducing a symmetry plane boundary condition. Typically, the relaxation zone should be long enough to allow for better generation and absorption of water waves. Nevertheless, it is very important to reduce the length of the overlap zone to reduce the size of the 3D model. Therefore, the length of the relaxation zone at the waves inlet side was tested for two ratios; $2.572 \mathrm{~L}$ (full 3D flume length) and $0.0273 \mathrm{~L}$ (reduced 3D domain) using theoretical Stokes fifth order waves of $\mathrm{T}=4 \mathrm{~s}$. and $\mathrm{H}=$ $0.8 \mathrm{~m}$. As shown in Figure 5, the moments exerted at the top of the pile are, relatively, in good agreement with physical experiments for both cases. For the short relaxation zone, the non-linearity effects of the generated 5th order Stokes wave are more apparent at the trough compared to waves generated by a long relaxation zone, which compares qualitatively better to measurements.

Both numerical solutions overestimate the inline moment amplitude, with the long relaxation zone giving relatively better results compared to measurements. The maximum inline moment exerted on a single pile is overestimated by about $10 \%$ when using a long relaxation zone and by about $36 \%$ when using a short relaxation zone. This result emphasizes the importance of providing a relatively long relaxation zone for the theoretical generation of water waves. Nevertheless, for the study at hand, the use of a short overlap (relaxation) zone for the 3D model is justified by the fact that the introduced wave train is retrieved from a CFD simulation and therefore needs less relaxation for introduction into the model to yield good results. The sampled water surface and wave kinematics from the 2D CFD model are used as input for the $3 \mathrm{D}$ reduced model via the hybrid $2 D 3 D$ extension module implemented in the waves 2 Foam toolbox.

Introducing highly nonlinear waves to the 3D domain with a short relaxation (overlapping) zone (or without it altogether) may cause the development of very high artificial fluid velocities, especially at the water-air interface near the end of the overlap zone (or near the inlet boundary). These artificial velocities cause the simulation to advance very slowly and eventually crash. It was found that applying underrelaxation of velocity to some cells based on an arbitrary threshold of an illogical value for velocity magnitude helps maintain solution stability and achieve faster convergence. The outlined procedure was applied only in a small region (of length $0.9 \mathrm{~m}$. along wave propagation in this study) just after the overlap (relaxation) zone, or just after the inlet boundary if no overlap zone is used. In the control region, the velocity magnitude is monitored for all cells. If the velocity magnitude in any cell crosses an (irrational) arbitrary value (e.g. $15 \mathrm{~m} / \mathrm{s}$ in this study), the velocity components in this cell are reduced by a percentage ( $90 \%$ in this study). It is necessary to ensure that the velocity relaxation (of selected cells) does not affect the integrity of the solution by considering a high enough value (that is indeed artificial) as a threshold of applying velocity relaxation and ensure non-aggressive relaxation. It was found that turbulence modelling plays a significant role regarding this problem, with RANS (k-w-SST) causing more convergence problems than LES or no turbulence modelling. It is worth mentioning that neither relaxing air velocity nor neglecting air phase convection can succeed in solving the artificial velocities problem. 


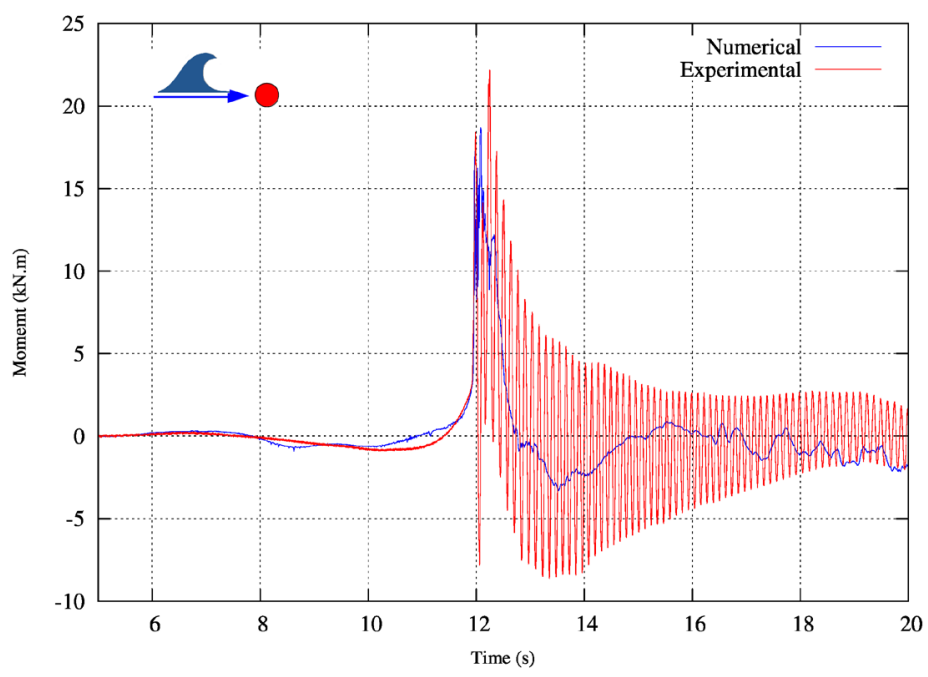

(a)

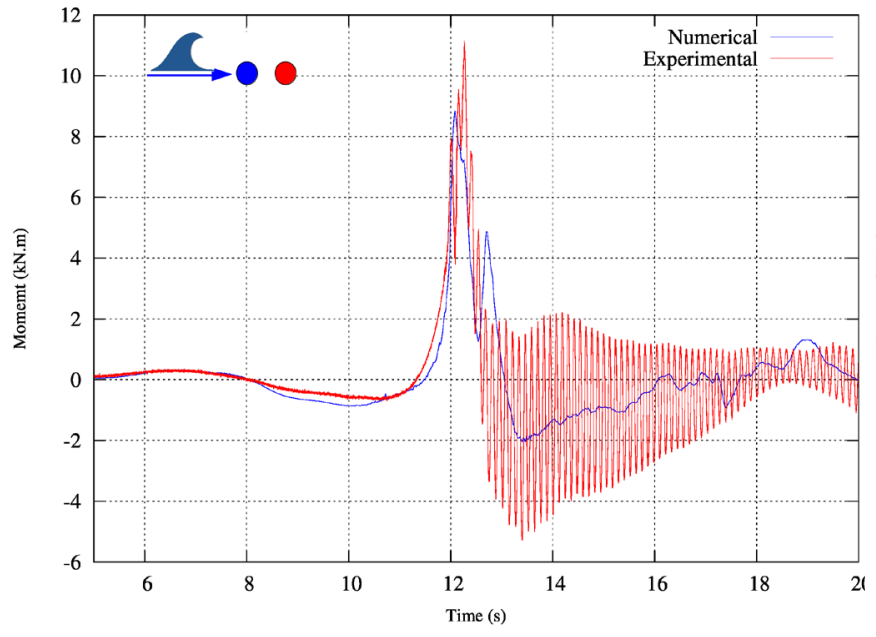

(b)

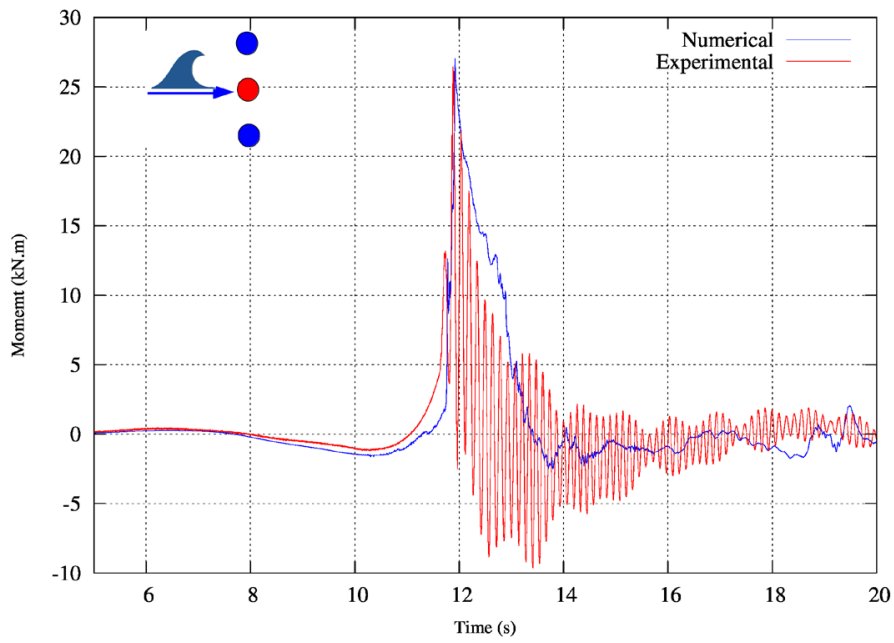

(c)

Figure 6. Inline moment on the top of (a) a single pile, (b) two piles in tandem and (c) three piles side by side for a Gaussian wave packet that focuses $103 \mathrm{~m}$. away from the wave maker (finest mesh size $0.01375 \mathrm{~m}$.)

The inline moment on top of the single pile due to impact of the focused-waves (from Figure 4), is shown in Figure 6. The numerical and experimental moments are relatively in good agreement. The maximum value of moment (at impact) exerted on the pile is well reproduced from the physical experiments.

The high frequency oscillation appearing in the numerically calculated moment (just after impact) is due to consideration of fluid compressibility that enhances the reproduction of wave impact. Nevertheless, a more apparent high frequency oscillation of higher amplitude is measured and is not reproduced numerically. This is most likely due to the pile structural response to the wave impact (vibration). The given interpretation becomes clear when one observes how the oscillation dampens after the impact. Basically, this response cannot be reproduced without considering a coupled CSD-CFD (Computational Structural Dynamics-Computational Fluid Dynamics) model system. Further, the pile vibration does not appear in experiments with regular waves as they seem to exert quasi-static loads on the pile.

Two cases of wave-pile group interaction are considered herein, as well, for validation of the model system: the case of two piles in tandem and three piles side by side (Figure 6). The approach of a focusedwave to the two piles in tandem is shown in Figure 7, with maps of the fluid velocity and pressure. The plan of the water surface elevation of the focused wave attacking two piles in tandem, for different times, is given in Figure 8. In this figure, the shedding of the wave by the front pile is evident, which yields the reduction of the forces exerted on the rear pile. The spacing (face to face) between piles for both cases is equal to the pile diameter. In forthcoming publications, the focus will be set on using the validated model system to enhance the understanding of the relevant hydrodynamic processes involved, and to extend the range of tested conditions in the laboratory 

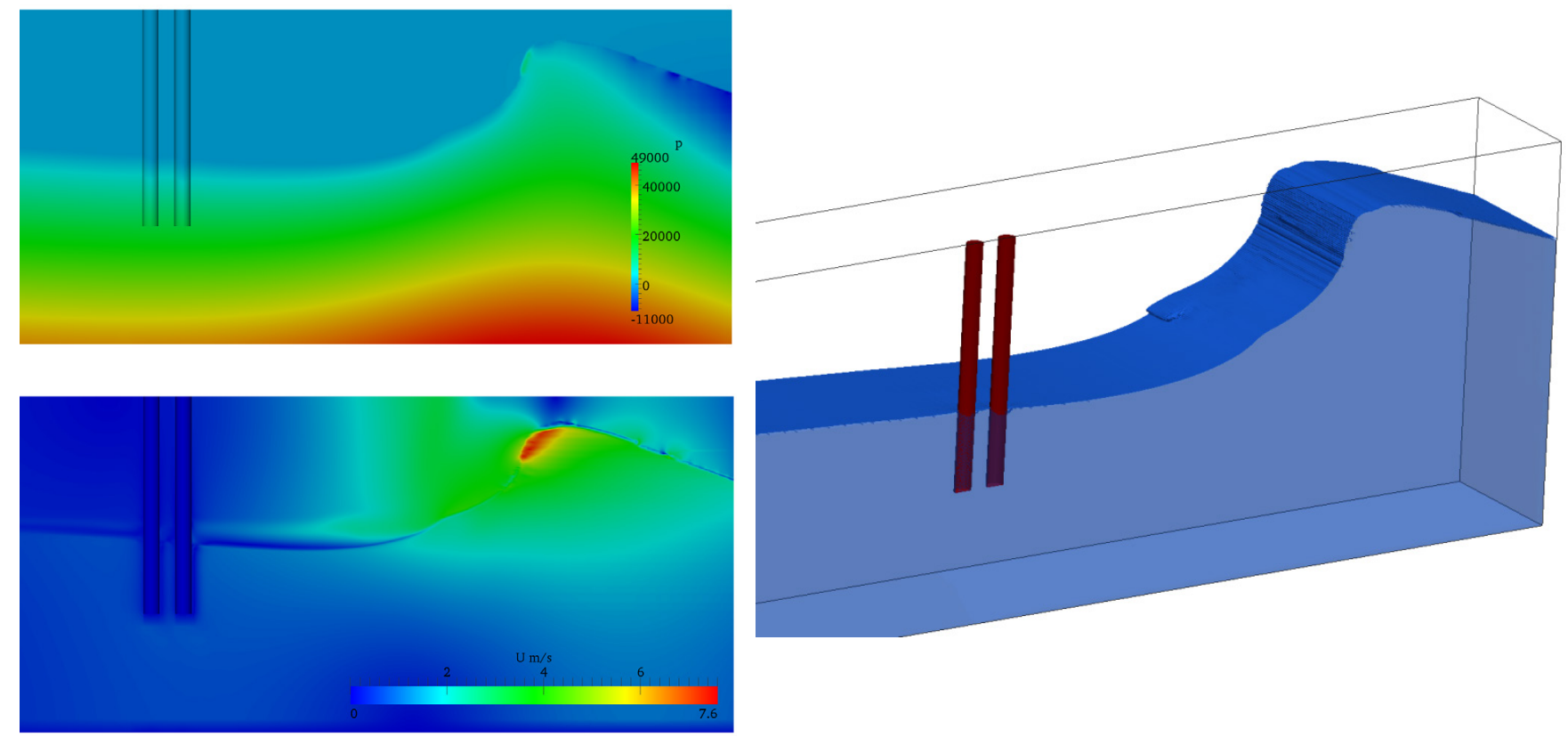

Figure 7. A focused wave approaching two piles in tandem (water surface, pressure and horizontal fluid velocity)
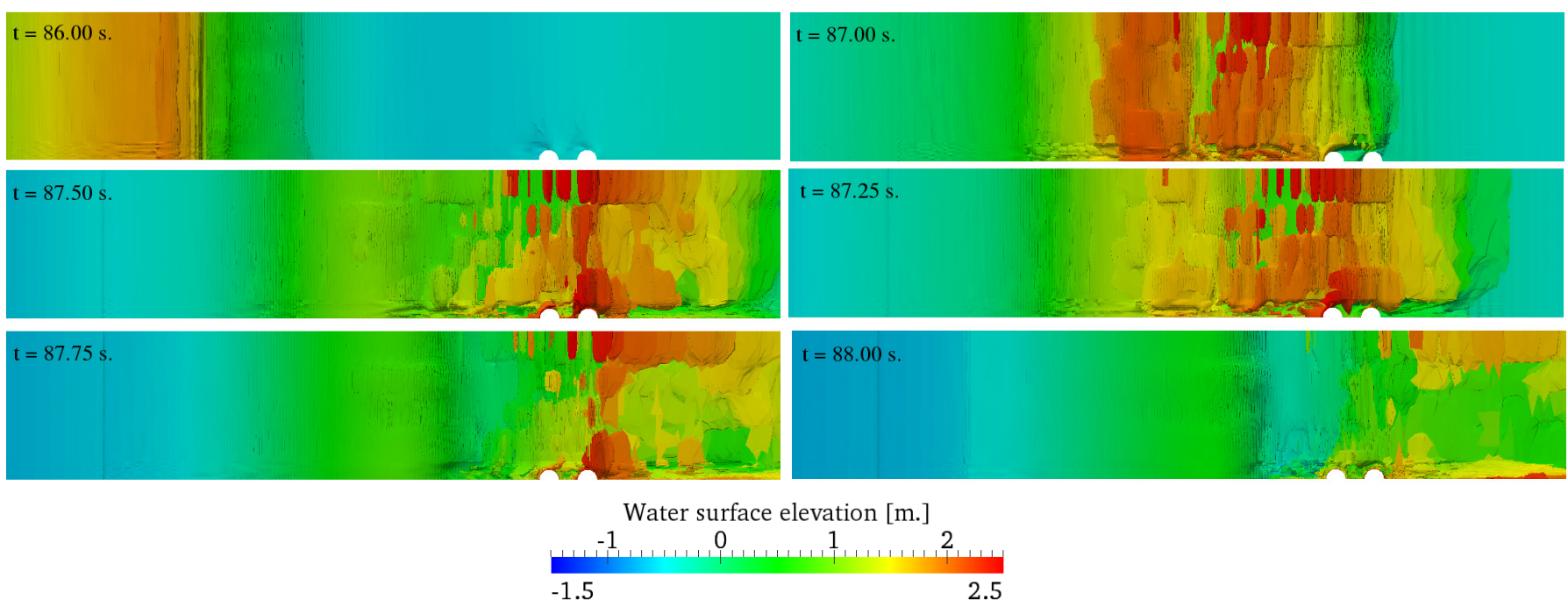

Figure 8. Plan of water surface elevation for a focused wave approaching two piles in tandem for different times

\section{SUMMARY AND CONCLUDING REMARKS}

A hybrid 2D-3D CFD model system is developed for the simulation of 3D wave loads on structures in the near field; where a reduced 3D domain is considered by using input data calculated from a 2D CFD model representing the far-field. The linking utilities make it trivial to couple any other far-field model with the reduced 3D domain. The concept is to test several structural configurations for the same wave conditions without redundancy in computations for the far field. The 2D CFD model applies dynamic mesh to model direct wave paddle motion similar to conditions in the physical experiments. The model system reduced the 3D domain size by $90 \%$ with a static mesh (less computational time). Further, the simulated time needed for waves to reach the structure in the $3 \mathrm{D}$ model is reduced appreciably (about $80 \%$ ). A procedure was presented for the control of development of artificial high velocities at the $3 \mathrm{D}$ domain to allow achievement (or enhancement) of convergence. Additionally, validation of the model system is presented. In forthcoming publications, the developed model system will be used for extending the testing conditions of the wave loads on a pile within a pile group. In addition, the model system will help to substantially improve the understanding of the problem. 


\section{ACKNOWLEDGEMENT}

The funding of the German Research Foundation (DFG, Deutsche Forschungsgemeinschaft) for the study through the WaPiGS project (Ou 1/13-1) and the HYBTEW Project (Ou 1/14-1) is gratefully acknowledged.

\section{NOTATION}

$\nabla \quad$ Nabla/del operator

U fluid velocity vector $[\mathrm{m} / \mathrm{s}]$

$p \quad$ fluid pressure $[\mathrm{Pa}]$

$K \quad$ bulk modulus $[\mathrm{Pa}]$

$\gamma \quad$ phase fraction

\section{REFERENCES}

Jacobsen, N. G., Fuhrman, D. R., and Fredsøe, J., 2012. "A Wave Generation Toolbox for the Open-Source CFD Library: OpenFoam ${ }^{\circledR}$ ". Int. J. Numerl. Meth. Fluids, 70(9), pp. 1073-1088.

Sparboom, U., and Oumeraci, H., 2006. "Wave loads of slender marine cylinders depending on interaction effects of adjacent cylinders". Proc. 25th OMAE, Hamburg, Germany, Paper No. 92626.

Bonakdar, L., and Oumeraci, H., 2012. "Interaction of waves and pile group-supported offshore structures: A large scale model study”. Proc. 22nd ISOPE, Rhodes, Greece. 